\title{
Reaction on a rink: Kondo-enhanced heterogeneous single-atom catalysis
}

Xiangyang Li, ${ }^{\dagger}$ Hong Gong, ${ }^{\dagger}$ Qingfeng Zhuang, ${ }^{\dagger}$ Bing Wang, ${ }^{\dagger}$ Xiao Zheng, ${ }^{*, \dagger}, \dagger$ and Jinlong Yang*,

$\dagger$ Hefei National Laboratory for Physical Sciences at the Microscale 83 Synergetic Innovation Center of Quantum Information and Quantum Physics, University of Science and Technology of China, Hefei, Anhui 230026, China

$\ddagger C A S$ Center for Excellence in Nanoscience $\& 3$ Key Laboratory of Surface and Interface Chemistry and Energy Catalysis of Anhui Higher Education Institutes, University of Science and Technology of China, Hefei, Anhui 230026, China

E-mail: xz58@ustc.edu.cn; jlyang@ustc.edu.cn 


\begin{abstract}
Boosting the efficiency of heterogeneous single-atom catalysts (SACs) by adjusting the microenvironment of the active atom has recently attracted enormous attention. However, it remains largely unknown whether the atomic spin could be utilized to enhance the catalytic activity. We explore such a possibility by investigating the thermochemical effect of Kondo screening of a local atomic spin by free electrons in the metal support. Inspired by the exothermicity of Kondo spin-screening, a novel approach to heterogeneous catalysis - reaction on a rink (ROAR) - is proposed. Interestingly, in contrast to the conventional notion of thermal catalytic reaction, lowering the temperature of metal support is predicted to result in a reduced reaction barrier. As a proof of concept, $\mathrm{CO}$ oxidation catalyzed by the $\mathrm{Co} @ \mathrm{CoPc} / \mathrm{Au}(111)$ composite is scrutinized and the efficacy of ROAR is demonstrated. This work accentuates the potential usefulness of the spin degrees of freedom to heterogeneous single-atom catalysis.
\end{abstract}

\title{
Graphical TOC Entry
}

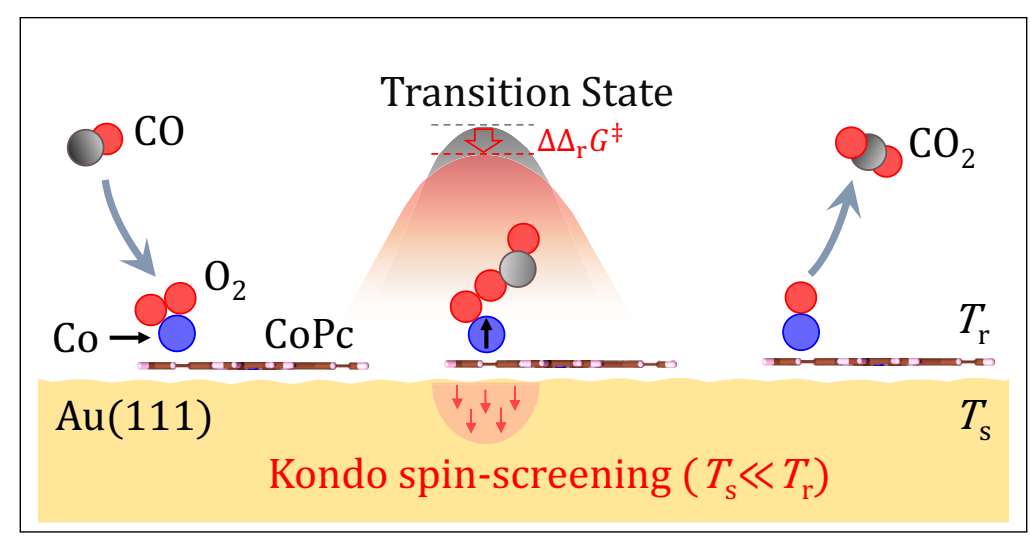




\section{Introduction}

For thermal catalytic reactions, one of the most important goals is to reduce the energy barrier of the rate-determining step, so that the reaction gets substantially accelerated and may occur under mild conditions. Recently, interests in heterogeneous single-atom catalysis have grown rapidly. ${ }^{1-3}$ This is not only because the atomically dispersed metal centers maximize the atom utilization efficiency, ${ }^{4-6}$ but also due to the remarkable catalytic activity and selectivity which have been demonstrated in experiments. ${ }^{7-9}$

It has been discovered that the spin states of metal atoms may have critical influence on the performance of single-atom catalysts (SACs). ${ }^{6,10-12}$ For instance, Liu et al. have reported that the intermediate-spin $\mathrm{FeN}_{5}$ species exhibits the highest activity and reusability among all the atomically dispersed Fe-N-C catalysts for the selective oxidation of $\mathrm{C}-\mathrm{H}$ bond. ${ }^{13}$ Sun et al. have found that one of the low-spin $\mathrm{FeN}_{4}-3 \mathrm{~N}$ isomers gives rise to a higher catalytic activity for oxygen reduction reaction than the pristine high-spin $\mathrm{FeN}_{4} \cdot{ }^{14}$ Moreover, Gong et al. have shown that, for the photocatalytic reduction of $\mathrm{CO}_{2}$ to $\mathrm{HCOOH}$, the covalent organic framework COF-367-Co ${ }^{\mathrm{III}}$ with the embedded cobalt ions in the $S=1 / 2$ state exhibits much higher activity and selectivity than the COF-367-Co ${ }^{\mathrm{II}}$ with $S=0$ cobalt ions. ${ }^{8}$ It is thus appealing to design practical schemes to enhance the catalytic performance by exploiting the spin degrees of freedom of SACs.

In recent years, Gracia et al. ${ }^{15-18}$ have explored the quantum spin exchange interaction (QSEI) between the electrons in the catalytic species and those in the reactant molecule and analyzed its nontrivial impact on the catalytic reactions. Physical and chemical means have been employed to modulate the QSEI, ${ }^{16,19,20}$ leading to enhanced electrocatalytic activity of certain magnetic materials, such as $\mathrm{CoFe}_{2} \mathrm{O}_{4}{ }^{19}$ and magnetized $\mathrm{Co}_{3-x} \mathrm{Fe}_{x} \mathrm{O}_{4} / \mathrm{Co}(\mathrm{Fe}) \mathrm{O}_{x} \mathrm{H}_{y} \cdot{ }^{20}$ Apart from this, various strategies have been proposed to optimize the atomic microenviron-

ment of SACs,${ }^{21}$ e.g. by utilizing the synergistic effects of neighboring atoms,${ }^{22-24}$ designing coordination ligands, ${ }^{25}$ tuning metal-support interaction, ${ }^{5,26}$ forming hydrogen bonding with surrounding atoms, ${ }^{27}$ etc. Despite the enormous efforts made, attempts to tune the spin-spin 
interaction between an SAC and its microenvironment have remained rather scarce. Some interesting questions can be raised, among which a fundamental one is: can the surrounding environment influence the local spin state of an SAC, and if so, can such influence be utilized to enhance the catalytic activity?

It is well-known that when a magnetic transition metal (TM) atom is adsorbed onto a metal support, the local spin moment on the TM adatom due to the spin-unpaired $d$-electrons can be screened by the itinerant electrons in the metal, provided that the temperature of metal support is low enough to suppress the thermal fluctuations of the free electrons. Such a phenomenon is referred to as the Kondo effect, ${ }^{28}$ which results in the formation of strongly correlated electronic states at the TM adatom/metal interface, as has been affirmed by the conspicuous zero-bias resonant peaks in the scanning tunneling spectroscopy. ${ }^{29}$ Extensive works have been carried out to fine-tune the strength of Kondo screening by adjusting the microenvironment of the TM adatom. ${ }^{30-34}$ However, the thermochemical change associated with the antiferromagnetic Kondo spin-screening phenomenon and its implication to the chemical reactions catalyzed by the TM adatom are largely unexplored.

The main goal of this work is to investigate the possibility of utilizing the local spin moment on the SAC and the Kondo correlation effect to enhance further the reactivity. To this end, we propose a novel approach to heterogeneous single-atom catalysis by harnessing the evolution of Kondo spin-screening interaction during the reaction process.

\section{Theory}

Figure 1 depicts the free energy profile of a prototypical heterogeneous reaction with an SAC loaded on a metal support. If the metal support has the same temperature as the reactant species, which is high enough to activate the thermal catalytic reaction, the spin orientations of the metal's free electrons are randomized by the strong thermal fluctuations. As a result, the averaged spin-screening effect is expected to be negligibly small. In contrast, 
if the temperature of metal support is low enough so that the thermal fluctuations of free electrons are largely suppressed, and meanwhile the magnetic orbital of the SAC couples strongly to the metallic states, the Kondo spin-screening will occur spontaneously.

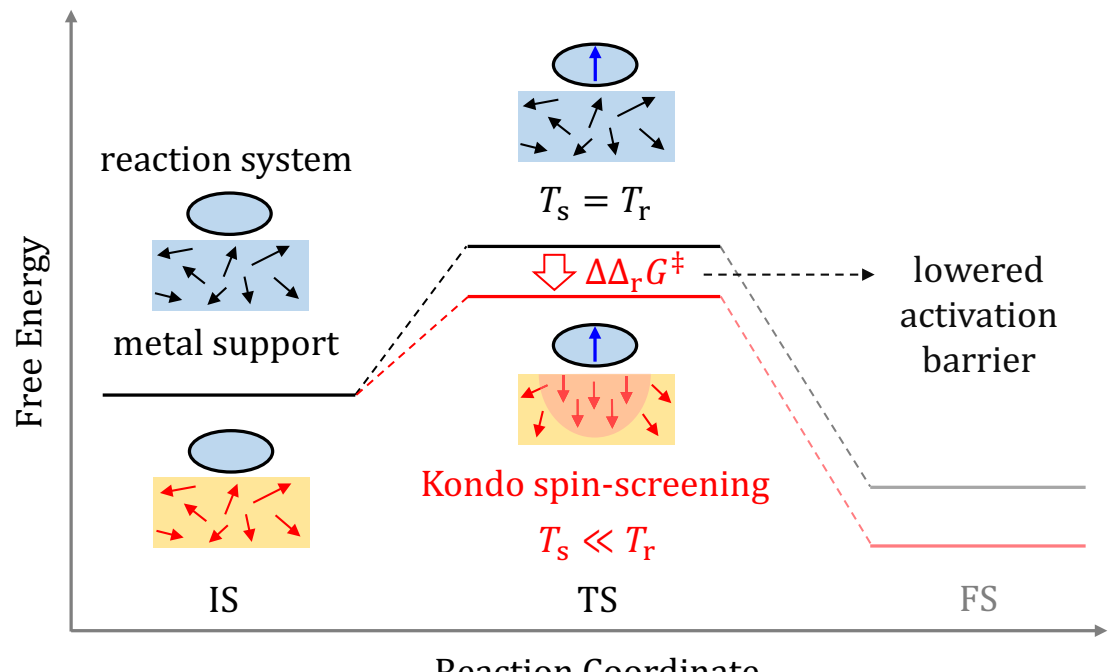

Figure 1: Schematic illustration of the proposed ROAR approach to heterogeneous singleatom catalysis. The horizontal line segments indicate the relative free energies of the composite reaction system (gaseous reactant species and metal support) in the initial state (IS), transition state (TS), and final state (FS). The solid arrows represent the electronic spins on the SAC and in the metal support. With the temperature of metal support $\left(T_{\mathrm{s}}\right)$ being much lower than that of the reactant species $\left(T_{\mathrm{r}}\right)$, Kondo states form at the $\mathrm{SAC} / \mathrm{metal}$ interface in the TS due to the spin-screening effect; see the main text for details. This leads to a reduction in the activation barrier $\left(\Delta \Delta_{\mathrm{r}} G^{+}\right)$, and hence could potentially accelerates the catalytic reaction.

From the above analysis, lowering the temperature of metal support $\left(T_{\mathrm{s}}\right)$ will result in a reduction of the activation barrier associated with the heterogeneous catalytic reaction, if the spin-screening effect presents only in the transition state (TS) but not in the initial state (IS). An ideal scenario is that the local spin moment on the SAC is appreciable in the TS but absent in the IS; see Figure 1. Such a scenario may be realized by a rational design of the SAC and its microenvironment. Experimentally, it has been shown that the Kondo spin-screening can be switched on and off by chemical doping. ${ }^{31-33}$

A reduced barrier height usually means enhanced kinetics. Thus, the heterogeneous reaction could be effectively accelerated by tuning $T_{\mathrm{s}}$ to be much lower than the temperature 
of reactant species $\left(T_{\mathrm{r}}\right)$, the accelerated heterogeneous reaction is reminiscent of skating on an ice rink. Therefore, we term the new approach to heterogeneous catalysis as "reaction on a rink" (ROAR).

Compared to the conventional situation of $T_{\mathrm{s}}=T_{\mathrm{r}}$, the activation free energy in a ROAR with $T_{\mathrm{s}} \ll T_{\mathrm{r}}$ is reduced by a certain amount, denoted by $\Delta \Delta_{\mathrm{r}} G^{+}$in Figure 1 . With the spins of free electrons aligning in an opposite direction to the local spin on the SAC, the spin configuration of the composite reaction system becomes more ordered, indicating a decrease in the entropy. Nevertheless, the screening of the local spin is an exothermic process because of the nonlocal electron correlation effect, and thus the formation of Kondo spin-screening is overall exergonic, i.e., $\Delta \Delta_{\mathrm{r}} G^{+}<0$.

By implementing the ROAR approach, the heterogeneous reaction system is far away from thermal equilibrium because of the appreciable temperature gradient across the gassolid interface. Since the temperature of the gaseous reactant species $\left(T_{\mathrm{r}}\right)$ is kept unchanged, the thermodynamic state and kinetic behavior of the gaseous species remain largely intact. At a greatly lowered substrate temperature $\left(T_{\mathrm{s}}\right)$, while the emerging Kondo spin-screening does not affect directly the atomic structure or charge state of the reactant species, it will give rise to a speedup factor $\gamma_{K}$ for the reaction rate as follows,

$$
\gamma_{\mathrm{K}} \simeq \exp \left(-\frac{\Delta \Delta_{\mathrm{r}} G^{\mp}}{R T_{\mathrm{r}}}\right)
$$

where $R$ is the gas constant. Here, we have assumed that the rate-determining step (RDS) is kinetically driven by the thermal energy of gaseous reactant species.

Based on Eq. (1), it is obvious that the speedup is more prominent with a more negative $\Delta \Delta_{\mathrm{r}} G^{\ddagger}$. The speedup gained via the ROAR is somewhat analogous to that achieved by harnessing the QSEI, ${ }^{16-18}$ since in both mechanisms the acceleration of the RDS originates from the reduced activation barrier due to generalized spin-exchange interactions. ${ }^{15,17}$ Nevertheless, a notable difference is that the QSEI involved in most of the previous works is 
ferromagnetic, while the Kondo spin-screening is antiferromagnetic in the ROAR.

By lowering the temperature of metal support from $T_{\mathrm{r}}$ to $T_{\mathrm{s}}$, the change in the vibrational entropy of adsorbed reactant species will also contribute to $\Delta \Delta_{\mathrm{r}} G^{\neq}$. In favorable situations, the variation of vibrational entropy could further enhance the speedup by ROAR.

To verify the practical feasibility of ROAR, in the following we propose theoretically a heterogeneous reaction which may be accelerated by the Kondo spin-screening effect. The reaction mechanism is scrutinized by carrying out first-principles-based calculations; see Section 3 for details.

\section{A potential candidate of ROAR - CO oxidation catalyzed by Co@CoPc/Au(111).} CO oxidation has been studied extensively in the field of heterogeneous catalysis. ${ }^{35-37}$ In particular, the use of single-atom and single-cluster catalysts has allowed $\mathrm{CO}$ oxidation to occur under low temperatures. ${ }^{38-40}$ For instance, the reaction temperature of $T_{\mathrm{r}}=200 \mathrm{~K}$ or even lower has been realized with dual-metal (Fe-Co) single sites dispersed on an N-doped carbon support, ${ }^{40} \mathrm{FeO}_{x}$ atomic layers deposited on a $\mathrm{Pt} / \mathrm{SiO}_{2}$ sample, ${ }^{38}$ and $\mathrm{Au}_{8}$ clusters bound to oxygen-vacancy F-center defects on an $\operatorname{Mg}(001)$ surface. ${ }^{39}$

Recently, we have successfully used cobalt phthalocyanine (CoPc) molecules as molds to regularize the local spin states of Co atoms dispersed on an $\mathrm{Au}(111)$ surface. ${ }^{30}$ It was found that a Co atom captured by a CoPc molecular mold locates either beneath or above an isoindole unit of the Pc ring, depending on how the composite system is prepared. In particular, when Co atoms are dispersed onto a self-assembled CoPc superlattice on an $\mathrm{Au}(111)$ surface, most of the Co atoms are located above the CoPc superlattice layer by chemisorption, and these exposed Co adatoms are potentially active SAC for CO oxidation. Moreover, the CoPc superlattice may serve as a heat insulator, which helps to keep $T_{\mathrm{s}}$ much lower than $T_{\mathrm{r}}$ during the reaction process and hence facilitates the realization of ROAR. In the following, we demonstrate that the catalytic activity of $\mathrm{Co} @ \mathrm{CoPc} / \mathrm{Au}(111)$ toward CO oxidation could be further enhanced by utilizing the ROAR approach. 


\section{Computational Methods}

A combined density functional theory (DFT) ${ }^{41,42}$ and hierarchical equations of motion (HEOM) ${ }^{43,44}$ approach is employed to carry out the first-principles-based calculations. The DFT $+\mathrm{HEOM}$ approach has been successfully applied to describe the Kondo spin-screening effect in magnetic atom adsorbed on the metal support. ${ }^{30,45,46}$

\section{Density functional calculation.}

The Perdew-Burke-Ernzerholf (PBE) generalized gradient approximation implemented in the Vienna ab initio simulation package ${ }^{47}$ is employed, with the DFT-D3 method of Grimme $^{48}$ used to improve the description of van der Waals interactions. We choose not to invoke the Hubbard- $U$ correction, ${ }^{49}$ because previous studies have indicated that the calculation results for the $\mathrm{Co} @ \mathrm{CoPc} / \mathrm{Au}(111)$ composite agree better with the experimental measurement $\left(d I / d V\right.$ spectra) in the absence of the Hubbard- $U$ correction. ${ }^{30,50}$ The projected augmented wave method is adopted with the energy cutoff of $400 \mathrm{eV}$. A vacuum space of $16 \AA$ is adopted in the $z$-direction to avoid the artificial interaction between mirror images of the composite system. For geometry optimizations, a slab model including three layers of $\mathrm{Au}$ atoms is used to represent the $\mathrm{Au}(111)$ support, with each layer containing $56 \mathrm{Au}$ atoms. The bottom two Au layers are fixed to preserve the structure of the bulk gold substrate, while all the other atoms are fully relaxed until the residual force on every atom is less than

$0.02 \mathrm{eV} / \AA .^{30,46}$ The convergence criterion for energy is set to $1 \times 10^{-5} \mathrm{eV}$. Because of the large size of supercell, the $\Gamma$-point approximation is adopted.

The adsorption energy $E_{\text {ads }}$ of molecules is defined as

$$
E_{\mathrm{ads}}=E_{\mathrm{mol} / \mathrm{s}}-E_{\mathrm{mol}}-E_{\mathrm{s}}
$$

where $E_{\mathrm{mol} / \mathrm{s}}, E_{\mathrm{mol}}$ and $E_{\mathrm{s}}$ are the energies of the composite adsorption system, an isolated gas molecule, and a clean $\mathrm{Au}(111)$ support, respectively.

The reaction pathway connecting the initial state (IS), transition state (TS) and final 
state (FS) is searched by using the climbing image nudged elastic band method, ${ }^{51}$ and the TS is settled with the force reduced below $0.05 \mathrm{eV} / \AA$. When the ROAR is implemented, the entire heterogeneous reaction system, including the gaseous molecules, the solid substrate, and the chemical species adsorbed on the substrate surface, is in a nonequilibrium state. To assess the speedup factor $\gamma_{\mathrm{K}}$, we consider the scenario that the reaction process reaches a steady state with a constant thermal gradient being preserved across the gas and solid phases. ${ }^{52}$ The metal support is usually a good heat conductor, and we further assume the gas molecules above the surface are subject to frequent intermolecular collisions. The bulk solid and gas phases are thus supposed to hold the constant temperatures $T_{\mathrm{s}}$ and $T_{\mathrm{r}}$, respectively. Consequently, the temperature gradient exists mostly at the solid/gas interface. To simplify the calculation, for the $\mathrm{CO}$ oxidation catalyzed by $\mathrm{Co} @ \mathrm{CoPc} / \mathrm{Au}(111)$, the composite $\mathrm{Co} @ \mathrm{CoPc} / \mathrm{Au}(111)$ is supposed to have a low temperature $T_{\mathrm{s}}$, while the adsorbed reactant and transition-state species are considered to have the high temperature $T_{\mathrm{r}}$. The contribution of vibrational entropy to the change of free energy barrier $\Delta \Delta_{\mathrm{r}} G^{*}$ is evaluated by taking into account such a temperature gradient; see Note S1 for details.

Anderson impurity model. A single-orbital $\mathrm{AIM}^{53}$ is adopted to represent the magnetic $d$ orbital on the Co atom (the impurity) and the $s$ orbitals in the gold support (the reservoir). The total Hamiltonian is comprised of three parts:

$$
H_{\mathrm{AIM}}=H_{\mathrm{imp}}+H_{\mathrm{s}}+H_{\mathrm{hyb}},
$$

where $H_{\mathrm{imp}}$ and $H_{\mathrm{s}}$ are the Hamiltonian of the magnetic impurity and the gold support, respectively; and $H_{\text {hyb }}$ describes the $s$ - $d$ hybridization.

For $\mathrm{CO}$ oxidation catalyzed by the $\mathrm{Co} @ \mathrm{CoPc} / \mathrm{Au}(111)$ composite, both the IS and TS involve a primary magnetic orbital. The magnetic impurity is described by

$$
H_{\mathrm{imp}}=\epsilon_{d}\left(\hat{n}_{\uparrow}+\hat{n}_{\downarrow}\right)+U \hat{n}_{\uparrow} \hat{n}_{\downarrow} .
$$


Here, $\hat{n}_{\sigma}=\hat{d}_{\sigma}^{\dagger} \hat{d}_{\sigma}$, where $\hat{d}_{\sigma}^{\dagger}\left(\hat{d}_{\sigma}\right)$ creates (annihilates) a spin- $\sigma$ electron $(\sigma=\uparrow$ or $\downarrow)$ on the magnetic $d$ orbital of energy $\epsilon_{d}$; and $U$ is the on-site electron-electron Coulomb repulsion energy. The substrate is modeled by a reservoir of noninteracting electrons, i.e., $H_{\mathrm{s}}=$ $\sum_{\sigma} \epsilon_{k} \hat{c}_{k \sigma}^{\dagger} \hat{c}_{k \sigma}$, where $\hat{c}_{k \sigma}^{\dagger}\left(\hat{c}_{k \sigma}\right)$ is the creation (annihilation) operator for a spin- $\sigma$ electron on the $k$ th metallic state. The impurity-reservoir hybridization has the form of $H_{\text {hyb }}=$ $\sum_{\sigma k} t_{k} \hat{c}_{k \sigma}^{\dagger} \hat{d}_{\sigma}+$ H.c., with $t_{k}$ being the coupling strength between the magnetic orbital and the $k$ th reservoir state.

The influence of electron reservoir on the magnetic impurity is fully characterized by the hybridization function, which assumes a Lorentzian form of $\Delta_{\mathrm{s}}(\omega) \equiv \pi \sum_{k}\left|t_{k}\right|^{2} \delta\left(\omega-\epsilon_{k}\right)=$ $\frac{\Gamma_{\mathrm{s}} W_{\mathrm{s}}^{2}}{\left(\omega-\Omega_{\mathrm{s}}\right)^{2}+W_{\mathrm{s}}^{2}}$. Here, $\Gamma_{\mathrm{s}}$ is the $s-d$ hybridization strength, and $\Omega_{\mathrm{s}}\left(W_{\mathrm{s}}\right)$ is the band center (width) of the electron reservoir. The energetic parameters of the AIM are extracted from the results of DFT calculations ${ }^{29,46,54}$ (in units of $\mathrm{eV}$ ): $\epsilon_{d}=-0.6, U=1.48, \Gamma_{\mathrm{s}}=0.14, \Omega_{\mathrm{s}}=0$, and $W_{\mathrm{s}}=5.5$. In particular, $\Gamma_{\mathrm{s}}$ is extracted from the Green's function of the magnetic $d$ orbital of the Co atom. Details are given in the Note S2.

Hierarchical equations of motion method. The HEOM method for fermionic environments $^{43,55}$ implemented in the HEOM-QUICK program ${ }^{44}$ is employed to quantitatively characterize the Kondo spin-screening effect in the single-orbital AIM. To ensure a high accuracy for the AIM with a low $T_{\mathrm{s}}$, a recently developed Fano spectrum decomposition scheme $^{56,57}$ is adopted to accurately unravel the reservoir correlation functions. The HEOM calculations are carried out with the truncation tier set to $L=4$.

The hybridization free energy in the TS is computed by using the thermodynamic integral formula: ${ }^{58}$

$$
G_{\mathrm{hyb}}^{\mathrm{TS}}=\int_{0}^{1} \frac{\mathrm{d} \lambda}{\lambda}\left\langle H_{\mathrm{hyb}}\right\rangle_{\lambda}
$$

where $\langle\cdots\rangle$ denotes the expectation value taken at the stationary state of the impurityreservoir composite with $H_{\text {hyb }}$ scaled by $\lambda$. Details are given in the Note S3 and Tables S1-S4. 


\section{Results and Discussion}

a
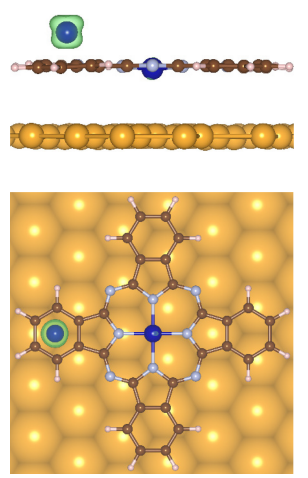

$\mathrm{Au}$ Co $\mathrm{N}$ C $\mathrm{H}$ 000

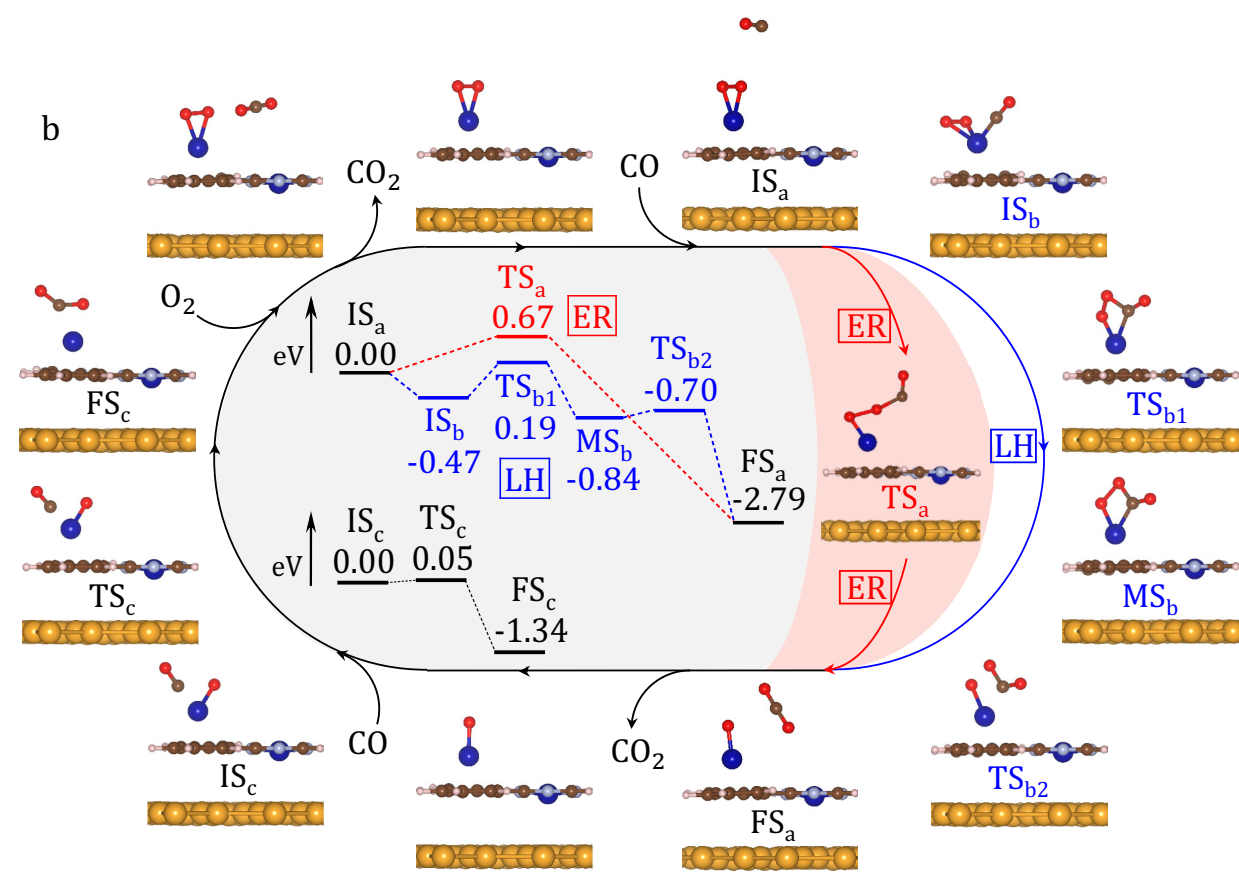

Figure 2: Reaction pathways for CO oxidation catalyzed by $\mathrm{Co} @ \mathrm{CoPc} / \mathrm{Au}(111)$. (a) Top and side views of a potential SAC - Co@CoPc adsorbed on an $\mathrm{Au}(111)$ surface. The green isosurface represents the spin density of $0.08 \AA^{-3}$. (b) Illustration of the complete catalytic cycles following the LH and ER mechanisms, with the corresponding potential energy profiles for the first and second $\mathrm{CO}$ oxidation steps depicted at the center of the panel.

Adsorption of $\mathrm{O}_{2}$ and $\mathrm{CO}$ on $\mathrm{Co} @ \mathbf{C o P c} / \mathbf{A u}(111)$. Figure 2(a) depicts the optimized geometric structure and spin density distribution of the $\mathrm{Co} @ \mathrm{CoPc} / \mathrm{Au}(111)$ composite system. The spin-unpaired electrons reside predominantly on the $d_{\pi}$ orbitals of the Co atom, and the dumbbell-shaped spin density distribution is a result of strong $d_{\pi}-\pi$ bonding between the Co atom and the nearby isoindole unit.

We then examine the adsorption of gaseous reactants onto an exposed Co atom and the associated thermochemical changes, as displayed in Figure 2(b). Calculation predicts an adsorption energy of $-2.63 \mathrm{eV}$ for a single $\mathrm{O}_{2}$, which is lower by $0.25 \mathrm{eV}$ than that for a single CO. This suggests that the Co@CoPc-catalyzed CO oxidation could proceed by following the Eley-Rideal (ER) mechanism. Besides, the energetically more favorable $\mathrm{O}_{2}$ adsorption 
effectively prevents the $\mathrm{CO}$ poisoning of the SAC. ${ }^{59}$

The co-adsorption of a $\mathrm{CO}$ onto the exposed Co atom with a pre-adsorbed $\mathrm{O}_{2}$ further stabilizes the whole composite system by $0.53 \mathrm{eV}$, and the subsequent $\mathrm{CO}$ oxidation occurs via the Langmuir-Hinshelwood ( $\mathrm{LH}$ ) pathway. While the co-adsorption of $\mathrm{CO}$ is barrierless in the most ideal scenario, in an actual co-adsorption process the attachment of a CO is likely to experience a finite energy barrier (see Figure S1), depending on the position and orientation of the $\mathrm{CO}$ as it approaches the Co atom.

Being a paramagnetic molecule, the adsorbed $\mathrm{O}_{2}$ partially quenches the local spin moment on the exposed Co atom. Moreover, the formation of the $\mathrm{O}-\mathrm{O}$ bond has a profound influence on the local electronic structure of the Co atom, which in turn varies significantly the strength of Kondo spin-screening.

Reaction pathways for CO oxidation and evolution of Kondo spin-screening. The $\mathrm{CO}$ oxidation catalyzed by $\mathrm{Co} @ \mathrm{CoPc} / \mathrm{Au}(111)$ could take both the ER and LH pathways. In either pathway, each adsorbed $\mathrm{O}_{2}$ oxidizes two $\mathrm{CO}$ molecules. The first oxidation is the $\mathrm{RDS}$, through which a $\mathrm{C}-\mathrm{O}$ bond is formed between one of the $\mathrm{O}$ atoms in the adsorbed $\mathrm{O}_{2}$ and a $\mathrm{CO}$; see Figures 2(b). Since both the adsorption of $\mathrm{O}_{2}$ and the desorption of the produced $\mathrm{CO}_{2}$ are barrierless, the $\mathrm{CO}$ oxidation is reaction-controlled. The RDS in both pathways have almost the same activation barrier heights. In the following, we will focus on the ER pathway, which is supposed to be more favorable because of the possible steric hindrance during the co-adsorption of CO.

Figure 3(a) depicts the energy profile of RDS in the ER pathway. Unlike the ideal scenario of Figure 1 where the IS is spin-unpolarized, here the Co atom in both IS and TS is in the $S=1 / 2$ local spin state; see the analysis of projected density of states (PDOS) of the Co $d$ orbitals in Table S5. Nevertheless, as will be elucidated below, the TS indeed has a much stronger spin-screening effect than the IS, which is critical to the realization of ROAR.

Kondo correlation depends crucially on the strength of hybridization $\left(\Gamma_{\mathrm{s}}\right)$ between the 


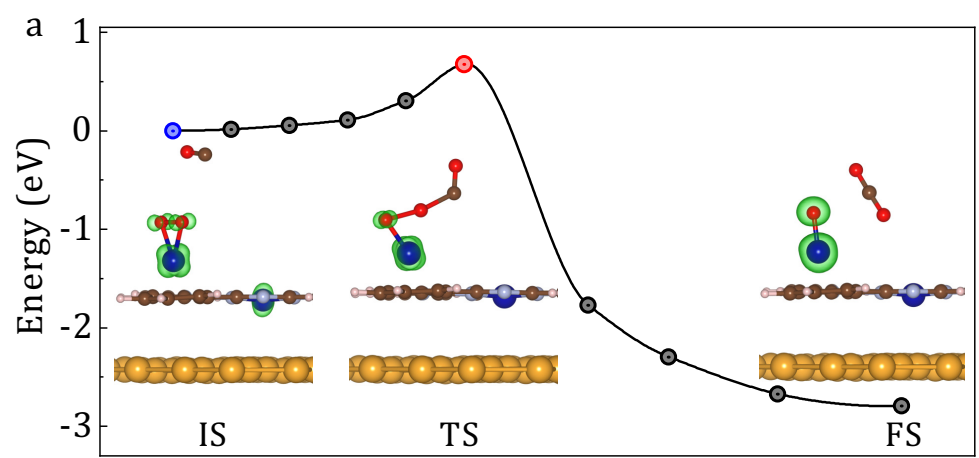

Reaction Coordinate

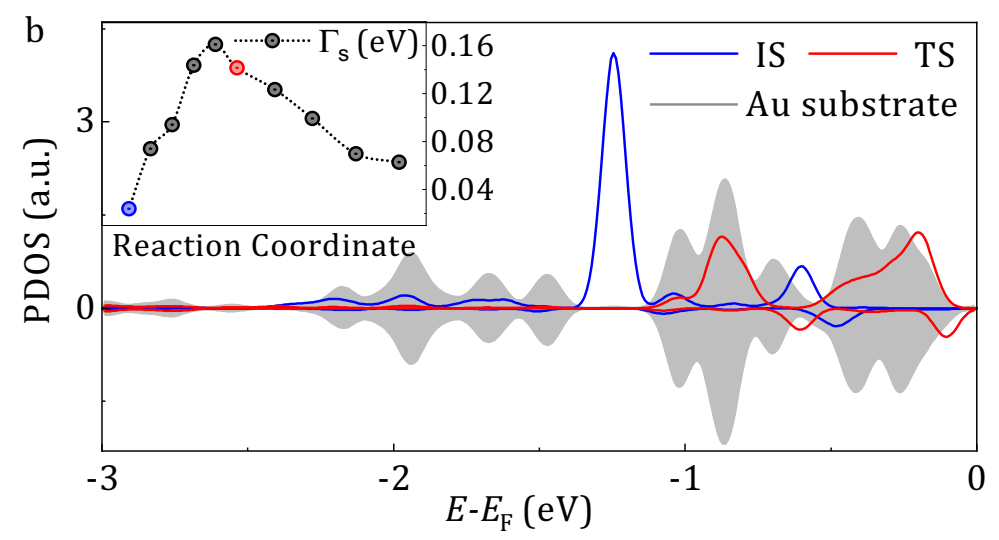

Figure 3: Evolution of Kondo spin-screening along the ER pathway of CO oxidation. (a) Potential energy profile of the first $\mathrm{CO}$ oxidation step. The green isosurfaces represent the spin density of $0.14 \AA^{-3}$ in the IS, TS and FS. (b) PDOS of the magnetic $d$ orbital on the Co atom $\left(d_{x z}\right.$ in the IS and $d_{y z}$ in the TS) and the $s$ orbitals of gold substrate, where positive (negative) values correspond to the majority-spin (minority-spin) electrons. The inset shows the variation of $s-d$ hybridization strength $\Gamma_{\mathrm{s}}$ along the reaction pathway. In particular, $\Gamma_{\mathrm{s}}=0.02 \mathrm{eV}$ and $0.14 \mathrm{eV}$ in the IS and TS, respectively.

magnetic $d$ orbital and the substrate metallic states. Regarding the PDOS shown in Figure 3(b), the main peaks of the magnetic $d$ orbital on the Co atom barely overlap those of the gold $s$ orbitals in the IS. In contrast, the overlap is rather conspicuous in the TS. This indicates a much stronger $s$ - $d$ hybridization in the TS than in the IS (see also Figure S2), which is further affirmed by the evolution of $\Gamma_{\mathrm{s}}$ displayed in the inset of Figure 3(b).

As a result of electron transfer from the gold substrate to the CoPc molecule, the central Co ion of the $\mathrm{CoPc} / \mathrm{Au}(111)$ composite is spin-unpolarized; see Table S5. Consequently, the Co ion may also adsorb CO molecules, ${ }^{60-62}$ and could thus affect the catalytic reaction of our interest. To this end, we examine explicitly the ER pathway with a CO molecule 
adsorbed on the central $\mathrm{Co}$ ion of the $\mathrm{CoPc} / \mathrm{Au}(111)$ composite. It is found that the surplus CO has a rather minor influence on the energy barrier (reduced by $0.01 \mathrm{eV}$ ) and the $s$ - $d$ hybridization strength (see Figure S3). This is because of the weak physisorption of CO at the central Co ion, which is similar to the previously reported $\mathrm{CO}$ adsorption onto the CoTPP $/ \operatorname{Ag}(111)$ composite. ${ }^{63}$ Moreover, the surplus $\mathrm{CO}$ is about $6 \AA$ away from the catalytic site (see Figure S4), and hence presents almost no steric hindrance to the reaction process.

Lowering of free energy barrier and acceleration of reaction rate with ROAR. We now explore the thermochemical change due to the prominent spin-screening effect in the TS and its consequence on the reaction kinetics. The activation barrier of RDS is $0.67 \mathrm{eV}$ under a normal reaction condition, which allows for a low reaction temperature of $T_{\mathrm{r}}=232 \mathrm{~K}$. Since the Co atom has a local spin of $S=1 / 2$ in the TS, a single-orbital Anderson impurity model (AIM) ${ }^{53}$ is employed to represent the $\mathrm{SAC} /$ metal composite, with which the magnetic $d$-orbital of Co and the metallic s-bands in the gold support are designated as the impurity and the reservoir, respectively.

The variation in activation barrier of RDS by implementing the ROAR approach can be evaluated by tracking the free energy change associated with the establishment of the $s$ - $d$ hybridization. Under the normal condition in which the metal support has a same temperature as that of the reactant species $\left(T_{\mathrm{r}}\right)$, the reaction energy barrier is expressed as

$$
\begin{aligned}
\Delta_{\mathrm{r}} G^{ \pm}= & G^{\mathrm{TS}}\left(T_{\mathrm{r}}\right)-G^{\mathrm{IS}}\left(T_{\mathrm{r}}\right) \\
= & {\left[G_{\mathrm{imp}}^{\mathrm{TS}}\left(T_{\mathrm{r}}\right)+G_{\mathrm{hyb}}^{\mathrm{TS}}\left(T_{\mathrm{r}}\right)+G_{\mathrm{s}}^{\mathrm{TS}}\left(T_{\mathrm{r}}\right)\right] } \\
& -\left[G_{\mathrm{imp}}^{\mathrm{IS}}\left(T_{\mathrm{r}}\right)+G_{\text {hyb }}^{\mathrm{IS}}\left(T_{\mathrm{r}}\right)+G_{\mathrm{s}}^{\mathrm{IS}}\left(T_{\mathrm{r}}\right)\right] \\
= & {\left[G_{\mathrm{imp}}^{\mathrm{TS}}\left(T_{\mathrm{r}}\right)-G_{\mathrm{imp}}^{\mathrm{IS}}\left(T_{\mathrm{r}}\right)\right]+\left[G_{\mathrm{hyb}}^{\mathrm{TS}}\left(T_{\mathrm{r}}\right)-G_{\mathrm{hyb}}^{\mathrm{IS}}\left(T_{\mathrm{r}}\right)\right] . }
\end{aligned}
$$

Here, $G_{\text {imp }}$ and $G_{\mathrm{s}}$ represent the Gibbs free energies of the isolated magnetic impurity and isolated reservoir, respectively; and $G_{\text {hyb }}$ denotes the free energy change before and after 


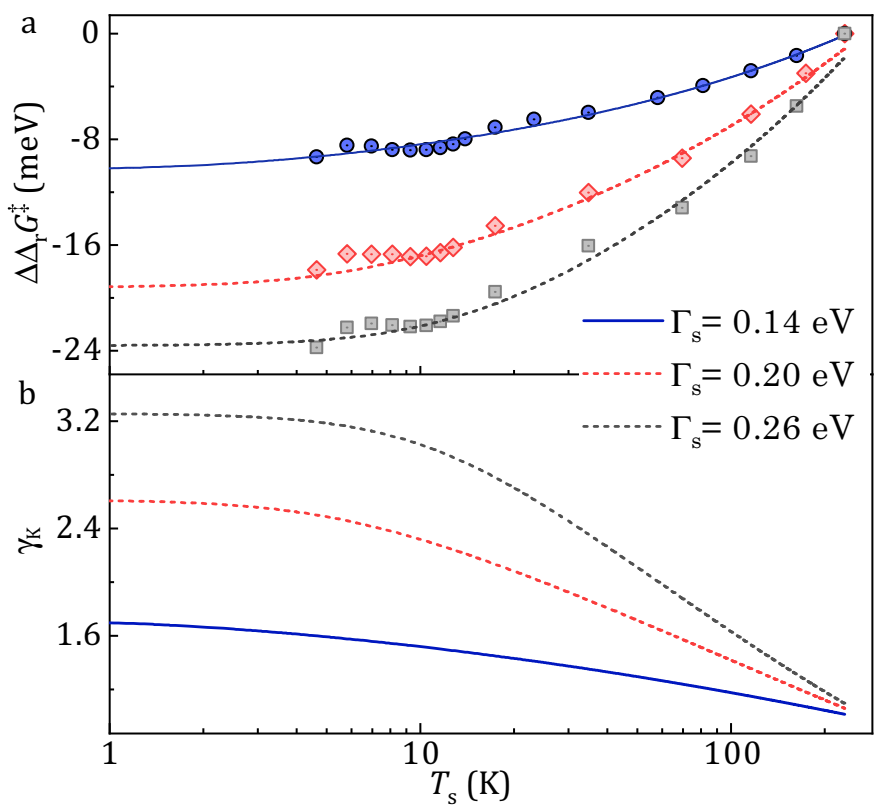

Figure 4: Lowering of activation barrier and acceleration of reaction due to Kondo spinscreening effect in the TS. (a) $\Delta \Delta_{\mathrm{r}} G^{\neq}$and (b) speedup factor $\gamma_{\mathrm{K}}$ versus $T_{\mathrm{s}}$, with $T_{\mathrm{r}}$ set to $232 \mathrm{~K}$. The scattered symbols in (a) represent the calculated data and the lines are fits to Eq. (9). The lines in (b) are the direct results of Eq. (1).

the $s$ - $d$ hybridization. While $G_{\mathrm{imp}}$ varies as the reaction proceeds, $G_{\mathrm{s}}$ can be regarded as a constant.

By implementing the ROAR, $T_{\mathrm{s}}$ is varied to be much lower than $T_{\mathrm{r}}$, and the free energy barrier becomes

$$
\begin{aligned}
\Delta_{\mathrm{ROAR}} G^{+}= & {\left[G_{\mathrm{imp}}^{\mathrm{TS}}\left(T_{\mathrm{r}}\right)+G_{\mathrm{hyb}}^{\mathrm{TS}}\left(T_{\mathrm{s}}\right)+G_{\mathrm{s}}^{\mathrm{TS}}\left(T_{\mathrm{s}}\right)\right] } \\
& -\left[G_{\mathrm{imp}}^{\mathrm{IS}}\left(T_{\mathrm{r}}\right)+G_{\mathrm{hyb}}^{\mathrm{IS}}\left(T_{\mathrm{s}}\right)+G_{\mathrm{s}}^{\mathrm{IS}}\left(T_{\mathrm{s}}\right)\right] \\
= & {\left[G_{\mathrm{imp}}^{\mathrm{TS}}\left(T_{\mathrm{r}}\right)-G_{\mathrm{imp}}^{\mathrm{IS}}\left(T_{\mathrm{r}}\right)\right] } \\
& +\left[G_{\mathrm{hyb}}^{\mathrm{TS}}\left(T_{\mathrm{s}}\right)-G_{\mathrm{hyb}}^{\mathrm{IS}}\left(T_{\mathrm{s}}\right)\right]
\end{aligned}
$$

Therefore, the reduction of free energy barrier by practising the ROAR is

$$
\Delta \Delta_{\mathrm{r}} G^{\neq} \equiv \Delta_{\mathrm{ROAR}} G^{\neq}-\Delta_{\mathrm{r}} G^{\neq} \simeq G_{\mathrm{hyb}}^{\mathrm{TS}}\left(T_{\mathrm{s}}\right)-G_{\mathrm{hyb}}^{\mathrm{TS}}\left(T_{\mathrm{r}}\right)
$$


Upon the last approximate equality of Eq. (8), we have used the fact that the $s$ - $d$ hybridization is rather weak in the IS, and thus $G_{\text {hyb }}^{\mathrm{IS}}\left(T_{\mathrm{s}}\right) \simeq G_{\text {hyb }}^{\mathrm{IS}}\left(T_{\mathrm{r}}\right)$.

It is interesting to find that the variation of $G_{\text {hyb }}^{\mathrm{TS}}$ with $T_{\mathrm{s}}$ fits accurately to an analytic formula of ${ }^{64}$

$$
G_{\mathrm{hyb}}^{\mathrm{TS}}\left(T_{\mathrm{s}}\right)=g_{a}\left[1+\left(\frac{T_{\mathrm{s}}}{T_{\mathrm{K}}}\right)^{2}\left(2^{\frac{1}{s}}-1\right)\right]^{s}+g_{b} .
$$

Here, $g_{a}$ and $g_{b}$ are two fitting parameters (see Table S6), $s=0.15$ is a constant, and $T_{\mathrm{K}}$ is an energy scale which characterizes the strength of Kondo correlation in the TS; see Note S4. ${ }^{65}$

As shown in Figure 4(a), $\Delta \Delta_{\mathrm{r}} G^{\neq}$becomes more negative with the lowering of $T_{\mathrm{s}}$, which results in an increasing speedup factor $\gamma_{\mathrm{K}}$. Specifically, $\Delta \Delta_{\mathrm{r}} G^{*}$ reaches $-0.011 \mathrm{eV}$ with $T_{\mathrm{s}}$ reduced from $232 \mathrm{~K}$ to $1 \mathrm{~K}$, and the emerging Kondo spin-screening leads to a speedup of the reaction rate by about 1.7 times. Although such an acceleration may seem minute, it is possible to enhance the efficacy of ROAR through rational design of the SAC and the metal support. For instance, if $\Gamma_{\mathrm{s}}$ in the TS could be enlarged to $0.20 \mathrm{eV}(0.26 \mathrm{eV})$, a more appreciable speedup of about 2.6 (3.3) times would be achieved; see Figure 4(b). In the literature, a $\Gamma_{\mathrm{s}}$ of $0.3 \mathrm{eV}$ has been reported for the $\mathrm{Co} / \mathrm{Cu}(100)$ composite. ${ }^{66}$

Admittedly, the kinetics of the overall heterogeneous catalytic reaction involves various microscopic process and is thus susceptible to many factors. Consequently, the speedup factor $\gamma_{\mathrm{K}}$ alone does not guarantee a conspicuous enhancement of the apparent reaction rate. Nevertheless, combined with some other favorable factors, the Kondo spin-screening effect may eventually lead to an appreciable acceleration of reaction rate via ROAR.

For the $\mathrm{CO}$ oxidation catalyzed by $\mathrm{Co} @ \mathrm{CoPc} / \mathrm{Au}(111)$, lowering $T_{\mathrm{s}}$ will not restrain the absorption/desorption rate of gaseous species to/from the surface, because both the adsorption of $\mathrm{O}_{2}$ and the desorption of $\mathrm{CO}_{2}$ are barrierless. Meanwhile, as long as $T_{\mathrm{r}}$ is kept constant, the thermodynamic state of gaseous reactants and their collision frequencies to the substrate surface will remain largely unchanged. ${ }^{67,68}$ Besides the Kondo spin-screening effect, the change in vibrational entropy of the adsorbate will also alter the activation free energy. 
Specifically, it is found that the free energy barrier of the RDS is further reduced by $0.057 \mathrm{eV}$ by including the influence of the vibration modes of the $\mathrm{Co} @ \mathrm{CoPc} / \mathrm{Au}(111)$ composite from $230 \mathrm{~K}$ to $1 \mathrm{~K}$; see Table S7. Such a reduction in energy barrier is even more pronounced than that resulted from the Kondo spin-screening effect.

It is well-known that the synergistic effect of microenvironment could further boost the catalytic efficiency of SACs. ${ }^{22-24}$ We have previously demonstrated that each adsorbed CoPc

molecule could uptake a maximum of four Co atoms, ${ }^{30}$ and the magnetic interactions between two Co adatoms, including the Ruderman-Kittel-Kasuya-Yosida (RKKY) interaction mediated by the Au s-bands and the spin-superexchange interaction mediated by the delocalized molecular orbital on the $\mathrm{CoPc}$, lead to an effective modulation of the local spin state of Co adatom and the strength of Kondo spin-screening. During the CO oxidation, while the superexchange channel is suppressed because the adsorbed $\mathrm{O}_{2}$ significantly impairs the delocalized molecular orbital, the RKKY interaction is preserved and is expected to give rise to an enhanced $\gamma_{\mathrm{K}}$. Interestingly, it is found that the RKKY interaction could lower the activation energy barrier of the RDS by as much as $0.039 \mathrm{eV}$; see Note S5, Table S8 and Figure S5. However, the theoretical determination of the enhancement of $\gamma_{\mathrm{K}}$ by the RKKY interaction is computationally too demanding with our present computer resources and is thus left for future investigation. Nontheless, the ROAR approach can be made even more favorable by exploiting the synergistic effect of the microenvironment.

\section{Conclusions}

To summarize, we have proposed a novel approach to heterogeneous catalysis - the ROAR, which aims at enhancing catalytic activity by exploiting the quantum correlation between the local spin on the SAC and the electronic spins in the metal support. As a proof of concept, $\mathrm{CO}$ oxidation catalyzed by the $\mathrm{Co} @ \mathrm{CoPc} / \mathrm{Au}(111)$ composite system is investigated. It is predicted that the ROAR will lead an appreciable acceleration of reaction rate. This work 
thus opens a new horizon for the field of heterogeneous single-atom catalysis by accentuating the potential usefulness of the spin degrees of freedom. In practice, since the ROAR favors an ultralow temperature of environment, it may be particularly valuable in outer space scenarios.

\section{Acknowledgement}

The support from the National Key Research and Development Program of China (Grant No. 2016YFA0200600), the National Natural Science Foundation of China (Grant Nos. 21973086 and 21688102), the Strategic Priority Research Program of Chinese Academy of Sciences (Grant No. XDB36000000), and the Anhui Initiative in Quantum Information Technologies (Grant No. AHY090000) is greatly appreciated. The computational resources are provided by the Supercomputing Center of University of Science and Technology of China and Tianjin Supercomputer Center.

\section{Supporting Information Available}

Details of computational methodology, influence of the RKKY interaction on energy barrier, co-adsorption of gas molecule on $\mathrm{Co} @ \mathrm{CoPc} / \mathrm{Au}(111)$, analysis on the $s$ - $d$ hybridization strength, analysis on the PDOS of central Co ion of CoPc, magnetic properties of Co adatom and fitting parameters for the free energy change, and reduction of free energy barrier by considering vibrational entropy correction. This material is available free of charge via the Internet at http://pubs.acs.org.

\section{References}

(1) Wang, A.; Li, J.; Zhang, T. Heterogeneous single-atom catalysis. Nat. Rev. Chem. 2018, 2, 65-81. 
(2) Lang, R.; Du, X.; Huang, Y.; Jiang, X.; Zhang, Q.; Guo, Y.; Liu, K.; Qiao, B.; Wang, A.; Zhang, T. Single-atom catalysts based on the metal-oxide interaction. Chem. Rev. 2020, 120, 11986-12043.

(3) Wang, L.; Chen, W.; Zhang, D.; Du, Y.; Amal, R.; Qiao, S.; Wu, J.; Yin, Z. Surface strategies for catalytic $\mathrm{CO}_{2}$ reduction: from two-dimensional materials to nanoclusters to single atoms. Chem. Soc. Rev. 2019, 48, 5310-5349.

(4) Li, X.; Yang, X.; Huang, Y.; Zhang, T.; Liu, B. Supported noble-metal single atoms for heterogeneous catalysis. Adv. Mater. 2019, 31, 1902031.

(5) Li, L.; Chang, X.; Lin, X.; Zhao, Z.-J.; Gong, J. Theoretical insights into single-atom catalysts. Chem. Soc. Rev. 2020, 49, 8156-8178.

(6) Wang, Y.; Su, H.; He, Y.; Li, L.; Zhu, S.; Shen, H.; Xie, P.; Fu, X.; Zhou, G.; Feng, C. et al. Advanced electrocatalysts with single-metal-atom active sites. Chem. Rev. 2020, 120, 12217-12314.

(7) Kaiser, S. K.; Fako, E.; Manzocchi, G.; Krumeich, F.; Hauert, R.; Clark, A. H.; Safonova, O. V.; López, N.; Pérez-Ramírez, J. Nanostructuring unlocks high performance of platinum single-atom catalysts for stable vinyl chloride production. Nat. Catal. 2020, 3, 376-385.

(8) Gong, Y.-N.; Zhong, W.; Li, Y.; Qiu, Y.; Zheng, L.; Jiang, J.; Jiang, H.-L. Regulating photocatalysis by spin-state manipulation of cobalt in covalent organic frameworks. $J$. Am. Chem. Soc. 2020, 142, 16723-16731.

(9) Sun, S.; Shen, G.; Jiang, J.; Mi, W.; Liu, X.; Pan, L.; Zhang, X.; Zou, J.-J. Boosting oxygen evolution kinetics by $\mathrm{Mn}-\mathrm{N}-\mathrm{C}$ motifs with tunable spin state for highly efficient solar-driven water splitting. Adv. Energy Mater. 2019, 9, 1901505. 
(10) Chen, P.; Tong, Y.; Wu, C.; Xie, Y. Surface/interfacial engineering of inorganic lowdimensional electrode materials for electrocatalysis. Acc. Chem. Res. 2018, 51, 28572866.

(11) Shen, G.; Zhang, R.; Pan, L.; Hou, F.; Zhao, Y.; Shen, Z.; Mi, W.; Shi, C.; Wang, Q.; Zhang, X. et al. Regulating the spin state of $\mathrm{Fe}^{\mathrm{III}}$ by atomically anchoring on ultrathin titanium dioxide for efficient oxygen evolution electrocatalysis. Angew. Chem. Int. Ed. 2020, 59, 2313-2317.

(12) Liu, J.-C.; Ma, X.-L.; Li, Y.; Wang, Y.-G.; Xiao, H.; Li, J. Heterogeneous Fe 3 singlecluster catalyst for ammonia synthesis via an associative mechanism. Nat. Commun. 2018, 9, 1-9.

(13) Liu, W.; Zhang, L.; Liu, X.; Liu, X.; Yang, X.; Miao, S.; Wang, W.; Wang, A.; Zhang, T. Discriminating catalytically active $\mathrm{FeN}_{x}$ species of atomically dispersed $\mathrm{Fe}-\mathrm{N}-\mathrm{C}$ catalyst for selective oxidation of the C-H bond. J. Am. Chem. Soc. 2017, 139, 1079010798.

(14) Sun, Y.; Sun, S.; Yang, H.; Xi, S.; Gracia, J.; Xu, Z. J. Spin-related electron transfer and orbital interactions in oxygen electrocatalysis. Adv. Mater. 2020, 32, 2003297.

(15) Gracia, J. Spin dependent interactions catalyse the oxygen electrochemistry. Phys. Chem. Chem. Phys. 2017, 19, 20451-20456.

(16) Munarriz, J.; Polo, V.; Gracia, J. On the role of ferromagnetic interactions in highly active Mo-based catalysts for ammonia synthesis. ChemPhysChem 2018, 103, 95739578.

(17) Gracia, J.; Sharpe, R.; Munarriz, J. Principles determining the activity of magnetic oxides for electron transfer reactions. J. Catal. 2018, 361, 331-338. 
(18) Gracia, J. Itinerant spins and bond lengths in oxide electrocatalysts for oxygen evolution and reduction reactions. J. Phys. Chem. C 2019, 123, 9967-9972.

(19) Wu, T.; Ren, X.; Sun, Y.; Sun, S.; Xian, G.; Scherer, G. G.; Fisher, A. C.; Mandler, D.; Ager, J. W.; Grimaud, A. et al. Spin pinning effect to reconstructed oxyhydroxide layer on ferromagnetic oxides for enhanced water oxidation. Nat. Commun. 2021, 12, 3634.

(20) Ren, X.; Wu, T.; Sun, Y.; Li, Y.; Xian, G.; Liu, X.; Shen, C.; Gracia, J.; Gao, H.J.; Yang, H. et al. Spin-polarized oxygen evolution reaction under magnetic field. Nat. Commun. 2021, 12, 2608.

(21) Li, X.; Liu, L.; Ren, X.; Gao, J.; Huang, Y.; Liu, B. Microenvironment modulation of single-atom catalysts and their roles in electrochemical energy conversion. Sci. Adv. 2020, 6, eabb6833.

(22) Ding, M.; Flaig, R. W.; Jiang, H.-L.; Yaghi, O. M. Carbon capture and conversion using metal-organic frameworks and MOF-based materials. Chem. Soc. Rev. 2019, 48, $2783-2828$.

(23) Ji, S.; Chen, Y.; Wang, X.; Zhang, Z.; Wang, D.; Li, Y. Chemical synthesis of single atomic site catalysts. Chem. Rev. 2020, 120, 11900-12314.

(24) Guo, X.; Gu, J.; Lin, S.; Zhang, S.; Chen, Z.; Huang, S. Tackling the activity and selectivity challenges of electrocatalysts toward the nitrogen reduction reaction via atomically dispersed biatom catalysts. J. Am. Chem. Soc. 2020, 142, 5709-5721.

(25) Qin, R.; Liu, K.; Wu, Q.; Zheng, N. Surface coordination chemistry of atomically dispersed metal catalysts. Chem. Rev. 2020, 120, 11810-11899.

(26) Liu, K.; Zhao, X.; Ren, G.; Yang, T.; Ren, Y.; Lee, A. F.; Su, Y.; Pan, X.; Zhang, J.; Chen, Z. et al. Strong metal-support interaction promoted scalable production of thermally stable single-atom catalysts. Nat. Commun. 2020, 11, 1-9. 
(27) Song, J.; Wei, C.; Huang, Z.-F.; Liu, C.; Zeng, L.; Wang, X.; Xu, Z. J. A review on fundamentals for designing oxygen evolution electrocatalysts. Chem. Soc. Rev. 2020, 49, 2196-2214.

(28) Kondo, J. Resistance minimum in dilute magnetic alloys. Prog. Theor. Phys. 1964, 32, 37.

(29) Zhao, A. D.; Li, Q. X.; Chen, L.; Xiang, H. J.; Wang, W. H.; Pan, S.; Wang, B.; Xiao, X. D.; Yang, J. L.; Hou, J. G. et al. Controlling the Kondo effect of an adsorbed magnetic ion through its chemical bonding. Science 2005, 309, 1542.

(30) Li, X.; Zhu, L.; Li, B.; Li, J.; Gao, P.; Yang, L.; Zhao, A.; Luo, Y.; Hou, J.; Zheng, X. et al. Molecular molds for regularizing Kondo states at atom/metal interfaces. Nat. Commun. 2020, 11, 2566.

(31) Liu, L. W.; Yang, K.; Jiang, Y. H.; Song, B. Q.; Xiao, W. D.; Li, L. F.; Zhou, H. T.; Wang, Y. L.; Du, S. X.; Ouyang, M. et al. Reversible single spin control of individual magnetic molecule by hydrogen atom adsorption. Sci. Rep. 2013, 3, 1210.

(32) Xie, L.; Lin, H.; Zhang, C.; Li, J.; Merino-Díez, N.; Friedrich, N.; Bouju, X.; Li, Y.; Pascual, J. I.; Xu, W. Switching the spin on a Ni trimer within a metal-organic motif by controlling the on-top bromine atom. ACS Nano 2019, 13, 9936-9943.

(33) Krull, C.; Robles, R.; Mugarza, A.; Gambardella, P. Site-and orbital-dependent charge donation and spin manipulation in electron-doped metal phthalocyanines. Nat. Mater. 2013, 12, 337-343.

(34) Jacob, D.; Kurth, S. Many-body spectral functions from steady state density functional theory. Nano Lett. 2018, 18, 2086-2090.

(35) Al Soubaihi, R. M.; Saoud, K. M.; Dutta, J. Critical review of low-temperature CO 
oxidation and hysteresis phenomenon on heterogeneous catalysts. Catalysts 2018, 8, 660.

(36) Ackermann, M.; Pedersen, T.; Hendriksen, B.; Robach, O.; Bobaru, S.; Popa, I.; Quiros, C.; Kim, H.; Hammer, B.; Ferrer, S. et al. Structure and reactivity of surface oxides on Pt(110) during catalytic CO oxidation. Phys. Rev. Lett. 2005, 95, 255505.

(37) Xie, X.; Li, Y.; Liu, Z.-Q.; Haruta, M.; Shen, W. Low-temperature oxidation of CO catalysed by $\mathrm{Co}_{3} \mathrm{O}_{4}$ nanorods. Nature 2009, 458, 746-749.

(38) Cao, L.; Liu, W.; Luo, Q.; Yin, R.; Wang, B.; Weissenrieder, J.; Soldemo, M.; Yan, H.; Lin, Y.; Sun, Z. et al. Atomically dispersed iron hydroxide anchored on Pt for preferential oxidation of $\mathrm{CO}$ in $\mathrm{H}_{2}$. Nature 2019, 565, 631-635.

(39) Yoon, B.; Häkkinen, H.; Landman, U.; Wörz, A. S.; Antonietti, J.-M.; Abbet, S.; Judai, K.; Heiz, U. Charging effects on bonding and catalyzed oxidation of CO on $\mathrm{Au}_{8}$ clusters on MgO. Science 2005, 307, 403-407.

(40) Wang, J.; You, R.; Zhao, C.; Zhang, W.; Liu, W.; Fu, X.-P.; Li, Y.; Zhou, F.; Zheng, X.; $\mathrm{Xu}, \mathrm{Q}$. et al. N-coordinated dual-metal single-site catalyst for low-temperature CO oxidation. ACS Catal. 2020, 10, 2754-2761.

(41) Kohn, W.; Sham, L. J. Self-consistent equations including exchange and correlation effects. Phys. Rev. 1965, 140, A1133.

(42) Hohenberg, P.; Kohn, W. Inhomogeneous electron gas. Phys. Rev. 1964, 136, B864.

(43) Jin, J. S.; Zheng, X.; ; Yan, Y. J. Exact dynamics of dissipative electronic systems and quantum transport: Hierarchical equations of motion approach. J. Chem. Phys. 2008, 128, 234703.

(44) Ye, L. Z.; Wang, X. L.; Hou, D.; Xu, R. X.; Zheng, X.; Yan, Y. J. HEOM-QUICK: 
a program for accurate, efficient, and universal characterization of strongly correlated quantum impurity systems. WIREs Comput. Mol. Sci. 2016, 6, 608.

(45) Wang, X. L.; Yang, L. Q.; Ye, L. Z.; Zheng, X.; Yan, Y. J. Precise control of local spin states in an adsorbed magnetic molecule with an STM tip: theoretical insights from first-principles-based simulation. J. Phys. Chem. Lett. 2018, 9, 2418.

(46) Wang, Y.; Zheng, X.; Li, B.; Yang, J. L. Understanding the Kondo resonance in the d-CoPc/Au(111) adsorption system. J. Chem. Phys. 2014, 141, 084713.

(47) Perdew, J. P.; Burke, K.; Ernzerhof, M. Generalized gradient approximation made simple. Phys. Rev. Lett. 1996, 77, 3865.

(48) Grimme, S.; Antony, J.; Ehrlich, S.; Krieg, H. A consistent and accurate ab initio parametrization of density functional dispersion correction (DFT-D) for the 94 elements H-Pu. J. Chem. Phys. 2010, 132, 154104.

(49) Liechtenstein, A.; Anisimov, V. I.; Zaanen, J. Density-functional theory and strong interactions: Orbital ordering in Mott-Hubbard insulators. Phys. Rev. B 1995, 52, R5467-R5471.

(50) Li, J. C.; Li, B.; Wang, Y.; Zhao, A. D.; Wang, B. Identifying site-dependent effects of an extra Co atom on electronic states of single Co-phthalocyanine molecule. J. Chem. Phys. 2015, 143, 034701.

(51) Henkelman, G.; Uberuaga, B. P.; Jónsson, H. A climbing image nudged elastic band method for finding saddle points and minimum energy paths. J. Chem. Phys. 2000, 113, 9901-9904.

(52) Yin, C.; Li, J.; Li, T.; Yu, Y.; Kong, Y.; Gao, P.; Peng, H.; Tong, L.; Zhang, J. Catalystfree synthesis of few-layer graphdiyne using a microwave-induced temperature gradient at a solid/liquid interface. Adv. Funct. Mater. 2020, 30, 2001396. 
(53) Anderson, P. W. Localized magnetic states in metals. Phys. Rev. 1961, 124, 41.

(54) Hou, D.; Wang, R. L.; Zheng, X.; Tong, N. H.; Wei, J. H.; Yan, Y. J. Hierarchical equations of motion for an impurity solver in dynamical mean-field theory. Phys. Rev. $B$ 2014, 90, 045141 .

(55) Li, Z. H.; Tong, N. H.; Zheng, X.; Hou, D.; Wei, J. H.; Hu, J.; Yan, Y. J. Hierarchical Liouville-space approach for accurate and universal characterization of quantum impurity systems. Phys. Rev. Lett. 2012, 109, 266403.

(56) Cui, L.; Zhang, H.-D.; Zheng, X.; Xu, R.-X.; Yan, Y. J. Highly efficient and accurate sum-over-poles expansion of Fermi and Bose functions at near zero temperatures: Fano spectrum decomposition scheme. J. Chem. Phys. 2019, 151, 024110.

(57) Zhang, H.-D.; Cui, L.; Gong, H.; Xu, R.-X.; Zheng, X.; Yan, Y. Hierarchical equations of motion method based on Fano spectrum decomposition for low temperature environments. J. Chem. Phys. 2020, 152, 064107.

(58) Gong, H.; Wang, Y.; Zhang, H.-D.; Qiao, Q.; Xu, R.-X.; Zheng, X.; Yan, Y. Equilibrium and transient thermodynamics: A unified dissipaton-space approach. J. Chem. Phys. 2020, 153, 154111.

(59) Li, Y.; Sun, Q. The superior catalytic CO oxidation capacity of a Cr-phthalocyanine porous sheet. Sci. Rep. 2014, 4, 4098.

(60) Hung, C.-H.; Peng, C.-H.; Shen, Y.-L.; Wang, S.-L.; Chuang, C.-H.; Lee, H. M. Preparation and oxygenation of cobalt N-confused porphyrin nitrosyl complexes. Eur. J. Inorg. Chem. 2008, 1196-1199.

(61) Gallagher, A. T.; Malliakas, C. D.; Harris, T. D. CO binding at a four-coordinate cobaltous porphyrin site in a metal-organic framework: Structural, EPR, and gas adsorption analysis. Inorg. Chem. 2017, 56, 4654-4661. 
(62) Bezzu, C. G.; Burt, L. A.; McMonagle, C. J.; Moggach, S. A.; Kariuki, B. M.; Allan, D. R.; Warren, M.; McKeown, N. B. Highly stable fullerene-based porous molecular crystals with open metal sites. Nat. Mater. 2019, 18, 740-745.

(63) Seufert, K.; Auwarter, W.; Barth, J. V. Discriminative response of surface-confined metalloporphyrin molecules to carbon and nitrogen monoxide. J. Am. Chem. Soc. 2010, 132, 18141-18146.

(64) Goldhaber-Gordon, D.; Göres, J.; Kastner, M.; Shtrikman, H.; Mahalu, D.; Meirav, U. From the Kondo regime to the mixed-valence regime in a single-electron transistor. Phys. Rev. Lett. 1998, 81, 5225.

(65) Žitko, R.; Bonča, J. Multiple-impurity Anderson model for quantum dots coupled in parallel. Phys. Rev. B 2006, 74, 045312.

(66) Choi, D.-J.; Rastei, M.; Simon, P.; Limot, L. Conductance-driven change of the Kondo effect in a single cobalt atom. Phys. Rev. Lett. 2012, 108, 266803.

(67) Lu, X.; Jin, D.; Wei, S.; Zhang, M.; Zhu, Q.; Shi, X.; Deng, Z.; Guo, W.; Shen, W. Competitive adsorption of a binary $\mathrm{CO}_{2}-\mathrm{CH}_{2}$ mixture in nanoporous carbons: Effects of edge-functionalization. Nanoscale 2015, 7, 1002-1012.

(68) Mu, B.; Li, F.; Huang, Y.; Walton, K. S. Breathing effects of $\mathrm{CO}_{2}$ adsorption on a flexible 3D lanthanide metal-organic framework. J. Mater. Chem. 2012, 22, 1017210178. 